\title{
Universal scaling laws of keyhole stability and porosity in 3D printing of metals
}

\section{Zhengtao Gan ( $\nabla$ zhengtao.gan@northwestern.edu )}

Northwestern University

\section{Orion Kafka}

Northwestern University

\section{Niranjan Parab}

Argonne National Laboratory https://orcid.org/0000-0002-3215-1466

\section{Cang Zhao}

Tsinghua University https://orcid.org/0000-0002-2464-2409

\section{Lichao Fang}

Northwestern University

\section{Olle Heinonen}

ANL https://orcid.org/0000-0002-3618-6092

\section{Tao Sun}

University of Virginia https://orcid.org/0000-0002-4881-9774

\section{Wing Kam Liu}

Northwestern University

\section{Article}

Keywords: Universal scaling laws, keyhole stability, porosity, 3D printing of metals

Posted Date: October 28th, 2020

DOI: https://doi.org/10.21203/rs.3.rs-94648/v1

License: (c) (1) This work is licensed under a Creative Commons Attribution 4.0 International License. Read Full License

Version of Record: A version of this preprint was published on April 22nd, 2021. See the published version at https://doi.org/10.1038/s41467-021-22704-0. 


\title{
Title: Universal scaling laws of keyhole stability and porosity in 3D printing of metals
}

\author{
Authors: Zhengtao Gan $^{1 *}$, Orion L. Kafka ${ }^{1 \dagger}$, Niranjan Parab ${ }^{2}$, Cang Zhao ${ }^{2}$, Lichao Fang ${ }^{1}$, Olle
} Heinonen $^{3}$, Tao Sun ${ }^{2 \#}$, Wing K. Liu ${ }^{1 *}$.

\section{Affiliations:}

${ }^{1}$ Department of Mechanical Engineering, Northwestern University, Evanston, IL 60208

${ }^{2}$ X-ray Science Division, Argonne National Laboratory, Lemont, IL 60439

${ }^{3}$ Materials Science Division, Argonne National Laboratory, Lemont, IL 60439

*Correspondence to: zhengtao.gan@ northwestern.edu (Z. Gan), w-liu@ northwestern.edu (W. K. Liu).

\#Current address: Department of Materials Science and Engineering, University of Virginia, Charlottesville, VA 22904

†Current address: Applied Chemicals and Materials Division, National Institute of Standards and Technology, Boulder, CO 80305

Abstract: We leverage ultrahigh-speed synchrotron x-ray imaging and high-fidelity multiphysics modeling to identify strikingly simple yet universal scaling laws for keyhole stability and porosity in metal three-dimensional (3D) printing. The laws apply broadly and remain accurate for different materials, processing conditions, and printing machines. We define

20 a new dimensionless number, the Keyhole number, to predict aspect ratio of a keyhole and the morphological transition from stable at low Keyhole number to chaotic at high Keyhole number. Furthermore, we discover inherent correlation between keyhole stability and porosity formation in metal 3D printing. By reducing the dimensions of the formulation of these challenging problems, the compact scaling laws will aid process optimization and defect elimination during metal 3D printing, and potentially lead to a quantitative predictive framework.

Main Text: In metal 3D printing, also called additive manufacturing, components are typically built layer-by-layer via local melting and (re)solidification of feedstock materials, often gasatomized metallic powders. This process provides considerable freedom to design local features, such as geometry and composition, in addition to enhancing manufacturing flexibility and

30 reducing material waste. However, metal 3D printing has a vast number of parameters with complex interactions and dependencies to be considered when making a component (1). Many authors have quantified the effects of various individual parameters or groups of parameters (26). However, universal physical relationships, which are proven to be valid for different materials, processing conditions, and machines, have remained elusive. The multivariate and multiphysics nature of the metal 3D printing processes complicates parameter optimization, materials development and selection, and real-time process control.

During laser powder-bed fusion 3D printing, a topological depression (termed a keyhole) frequently forms, which is caused by vaporization-induced recoil pressure (7). Keyhole dynamics is inherently difficult to understand and predict because of its complex dependence upon many physical mechanisms, but important to be able to quantify because it is highly related 
to energy absorption and defect formation in metal 3D printing. The geometry of the keyhole significantly affects the energy coupling mechanisms between the high-power laser and the material (3), which leads to unusual melt pool dynamics (8) and solidification defects (1). An instable keyhole might also cause severe process instability and structural defects including porosity, balling effect, spattering, and unusual microstructural phases (9). A recent research capturing meso-nanosecond keyhole dynamics with high-fidelity simulations discovered keyhole-induced back-spattering and frozen depression defects (10). Although such keyholes were studied for laser welding in 1970s (11), high-quality in-situ experimental data on keyhole dynamics became available via high-speed x-ray imaging only recently (12-15). As compared with traditional post-mortem characterization of cross-sectioned fusion regions (3-5), ultrahighspeed $\mathrm{x}$-ray imaging provides adequate temporal and spatial resolutions for probing keyhole evolution and stability.

Another longstanding issue in metal 3D printing and welding is the generation of excessive porosity. Much effort has been directed at determining the physics underlying this phenomenon, as well as finding ways to eliminate or ameliorate it (16). Several mechanisms that lead to porosity formation have been identified, such as lack of fusion (17), instability of the depression zone (6), vaporization of volatile elements (18) and hydrogen precipitation (19). However, these efforts are still far from producing a predictive model for porosity - it is challenging to distinguish the quantitative impact of different mechanisms on the final, observed porosity. This makes it impossible to predict the porosity type and magnitude, and to optimize processing conditions to build pore-free parts. Elegant insights into the behavior of complex systems, such as metal 3D printing, can be provided by low-dimensional patterns expressed as compact mathematical equations or scaling laws. This adds simplicity to highly multivariate and/or multi-dimensional systems and helps guide process tailoring towards rapid scientific discovery and optimum engineering design (20).

In order to identify scaling laws in 3D printing, we start by generating and collecting insitu synchrotron $x$-ray imaging (2) data with various process parameters and materials (Data S1). We then apply dimensional analysis (21) to normalized governing equations of the system to identify scaled parameters and achieve dimensionality reduction. A multiphysics model is used to interpret energy absorption mechanisms and support the scaling laws. Those compact scaling laws with the property of dimensional homogeneity can be confirmed via the application of nonlinear symbolic regression method such as genetic programming (22).

The aspect ratio of the keyhole, $\mathrm{e}^{*}$, defined as keyhole depth $e$ divided by laser spot radius $r_{0}$ (Fig. S1), is a critical parameter in laser manufacturing processes, because it can be used to classify the laser-metal interaction into conduction, transition, and keyhole modes (7). The keyhole aspect ratio in a process is determined by the process parameters and material characteristics. The keyhole depth is directly quantified from in-situ X-ray images in this study, while most of literature measures the depth post-facto from cross-sections of the fusion region (3-5). We found that both the keyhole aspect ratio and its fluctuation exhibit universal scaling 40 with only one dimensionless parameter (Fig. 1A), thus we define this dimensionless parameter as the Keyhole number Ke,

$$
\mathrm{Ke}=\frac{\eta P}{\left(T_{l}-T_{0}\right) \pi \rho C_{p} \sqrt{\alpha V_{s} r_{0}^{3}}}
$$


which is defined by the absorptivity $\eta$, laser power $P$, liquidus temperature $T_{l}$, substrate temperature $T_{0}$, density $\rho$, heat capacity $C_{p}$, thermal diffusivity $\alpha$, scan speed $V_{s}$, and laser radius $r_{0}$. The form of Keyhole number can be derived from heat transfer theory with simplifying assumptions (Supplementary Text). There is a nearly linear relationship between the keyhole aspect ratio $\mathrm{e}^{*}$ and $\mathrm{Ke}$ (shown by the dashed line in Fig. 1A):

$$
\mathrm{e}^{*}=0.4(\mathrm{Ke}-1.4)
$$
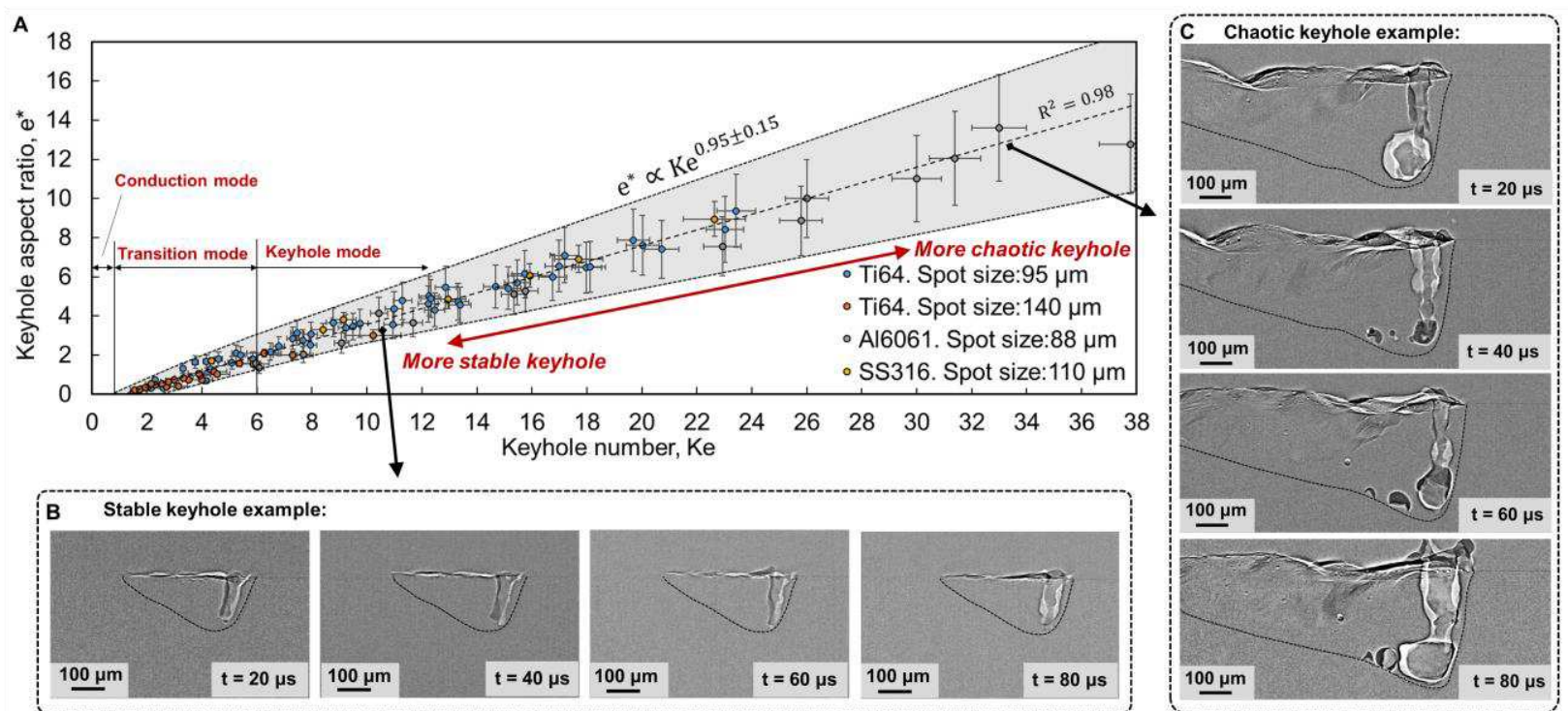

Fig. 1. One-dimensional law for keyhole aspect ratio controlled by the Keyhole number. (A) Identified scaling law, which is universal because all the data collapse to a single curve, even though they correspond to various laser power $(100-520 \mathrm{~W})$, scan speed $(0.3-1.2 \mathrm{~m} / \mathrm{s}$ ), laser spot size $(44-70 \mu \mathrm{m}$ ), and three different materials (Ti-6Al-4V (Ti64) (2), Aluminum alloy 6061 (A16061) and Stainless Steel 316 (SS316)). Time-dependent keyhole depth is measured from high-speed x-ray images at a $20 \mu \mathrm{s}$ interval for each process condition. The maximum and minimum of keyhole aspect ratio during the time period when the laser scans $2 \mathrm{~mm}$ length at the middle of the sample are marked as vertical error bars. Horizonal error bars account for uncertainties of the process parameters and material properties. (B) Operando X-ray image series with a $20 \mu$ s interval showing the keyhole and melt pool morphologies at stable keyhole region of Al6061. Laser power is $416 \mathrm{~W}$ and scan speed is $0.6 \mathrm{~m} / \mathrm{s}$. (C) Operando x-ray images showing keyhole morphologies at chaotic keyhole region of Al6061. Laser power is $520 \mathrm{~W}$ and scan speed is $0.3 \mathrm{~m} / \mathrm{s}$. Fusion boundary (outlined by the black dashed line) can be identified by the x-ray imaging because of the density difference inside and outside the fusion region. Although the x-ray experiments are conducted on a bare plate, a previous study reports that the keyhole depth does not change significantly by adding a thin layer of powder (2).

The identified scaling law for keyhole aspect ratio (or keyhole depth) is different from the law for fusion region depth (or melt pool depth) (3). The depth of the fusion region is certainly larger than the keyhole depth and does not linearly scale with keyhole depth owing to the drastic difference in melt pool geometry and melt flow pattern under different laser conditions. We can thus quantify the three different melting modes of the material using the keyhole aspect ratio $\mathrm{e}^{*}$ and Keyhole number Ke: conduction mode $\left(\mathrm{e}^{*}=0\right.$ or Ke $\left.<1.4\right)$, transition mode $\left(\mathrm{e}^{*} \leq\right.$ 2 or $1.4 \leq \mathrm{Ke} \leq 6.0)$, and keyhole mode $\left(\mathrm{e}^{*}>2\right.$ or Ke $\left.>6.0\right)$. We can also show the scaling behavior of the keyhole aspect ratio $\mathrm{e}^{*}$ :

$$
\begin{gathered}
\mathrm{e}^{*} \propto \eta P V_{s}^{-\frac{1}{2}} r_{0}{ }^{-\frac{2}{3}}, \\
\mathrm{e}^{*} \propto\left(T_{l}-T_{0}\right)^{-1}\left(\pi \rho C_{p}\right)^{-1} \alpha^{-\frac{1}{2}}
\end{gathered}
$$

Equation 3 represents a scaling law with respect to process parameters. It indicates that the keyhole aspect ratio is proportional to absorbed laser power $\eta P$ and has a power-law 
dependence on scan speed $V_{s}$ and laser spot radius $r_{0}$. The scaling order (exponent) is $-\frac{1}{2}$ for $V_{s}$ and $-\frac{2}{3}$ for $r_{0}$, which quantifies the effective strength of each process parameter on keyhole aspect ratio. From Equation 3, we can also argue that the traditionally used parameters, including linear energy density $\frac{P}{V_{S}}$, surface energy density $\frac{P}{V_{S} r_{0}}$, and volumetric energy density $\frac{P}{V_{S} r_{0}{ }^{2}}$ are not appropriate scaling parameters for the keyhole aspect ratio (Fig. S2). Equation 4 scales the effect of material attributes, which is useful for understanding and improving the printability of metals. This scaling law explains why printing light alloys with lower liquidus temperature $T_{l}$ and density $\rho$ but much higher thermal diffusivity, such as aluminum or magnesium alloys, tends to create a lower keyhole aspect ratio under the same process conditions when compared to, for example, steel.

More interestingly, the variation in the keyhole aspect ratio, $\Delta \mathrm{e}^{*}$, during the process represents the fluctuation and instability of the melt pool dynamics (Fig. 1 and Movie S1-S11). The melt pool instability could lead anomalies and structural defects, such as porosity, spattering, and high surface roughness. The shadowed region in Fig. 1A represents the variation in the keyhole aspect ratio $\Delta \mathrm{e}^{*}$, which approximately scales with the Keyhole number as

$$
\Delta \mathrm{e}^{*}=0.36 \mathrm{Ke}^{0.86} \text {. }
$$

We emphasize that the keyhole instability or variability is not observable using the traditional post-mortem characterization (3-5). We discover a scaling law for the variation in the keyhole aspect ratio (Equation 5) using x-ray imaging. When the Keyhole number is relatively small, e.g., $\mathrm{Ke}<16$, the keyhole fluctuation is small and thus there is almost no porosity and spattering events are rare (Fig. 1B). However, when the Keyhole number is large, e.g., Ke > 30, the keyhole exhibits chaotic features and becomes unstable (Fig. 1C). A small perturbance will lead to a huge fluctuation of the keyhole wall, and consequently pore and spatter generation. This discovery provides a clear guideline for designing the parameter space to achieve a stable 25 keyhole.

The absorptivity $\eta$ used in the expression of the Keyhole number is coupled with the keyhole morphology (7). To provide insight on the energy absorption and quantify the effects of process parameters and materials, we use a high-fidelity multiphysics model that is validated with operando high-speed x-ray imaging experiments (Fig. 2). The model simulates 3D temperature, velocity, pressure and vapor chemical species fields with $2 \mu \mathrm{m}$ resolution and is predictive because it uses laser ray-tracing method to calculate laser absorption and reflection within the keyhole (Fig. S4). Furthermore, the model accounts for evaporation-induced vapor plume, which is not considered in the most multiphysics models $(3,6,10)$ (Supplementary Text). The multiphase fluid mechanics is coupled with heat transfer solved on a 10-ns time scale to accurately capture laser-keyhole and keyhole-vapor interactions. The model does not consider plasma generation and laser attenuation caused by the vapor plume or plasma. 


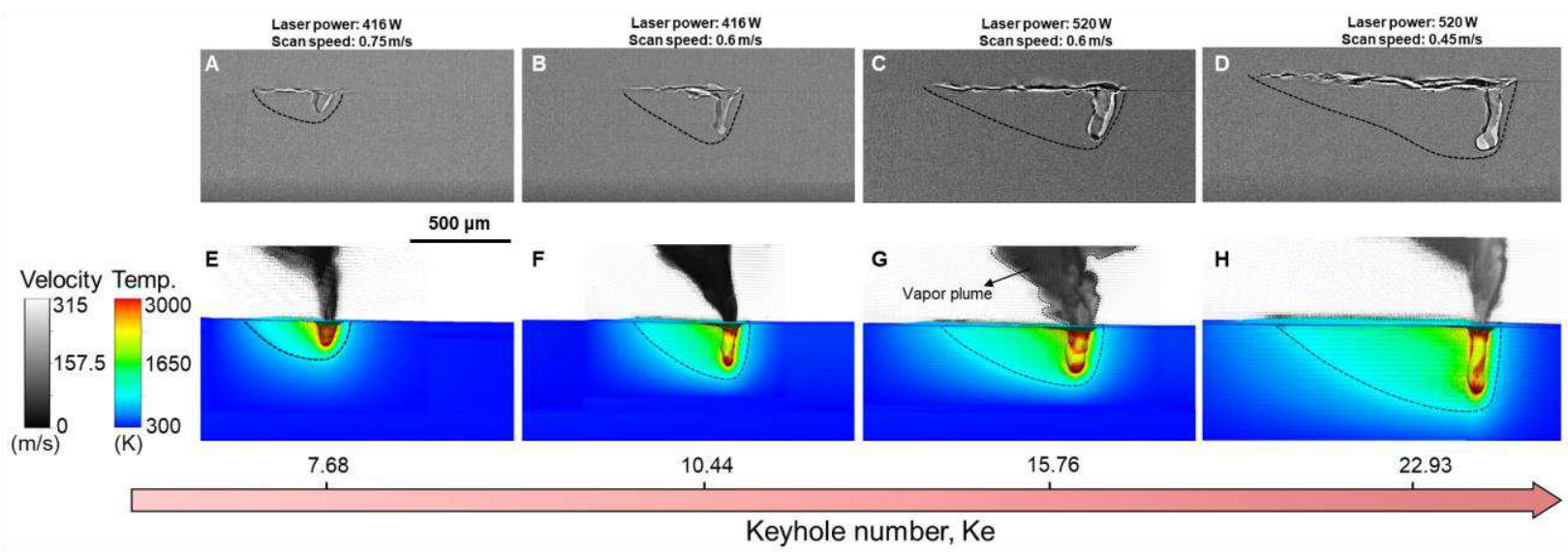

Fig. 2. Comparison between experimental data and multiphysics simulations with various Keyhole number. (A-D) Operando x-ray images of the scanning laser melting of Al6061 at steady state. (E-H) Multiphysics modeling showing temperature contour at the longitudinal cross-section and arrow field of vapor velocity above the substrate (the length of the arrow is proportional to the magnitude of velocity at the specific point). The simulated high-speed vapor jet ( $200 \mathrm{~m} / \mathrm{s})$ impacts keyhole interface which is one of the sources driving the fluctuation of the keyhole and energy loss due to convection.

To select dominant effects and parameters for the scaling law, our model analyzes, for the first time, energy losses due to various mechanisms including the reflection of the laser beam, convection caused by the vapor jet, latent heat of evaporation, surface radiation, and droplet spattering (definitions in Supplementary Text). The absorbed energy equals the laser energy input minus all the energy losses. Since the absorbed energy varies in a complex way depending on the keyhole morphology and temperature, our multiphysics model captures the transient keyhole morphology and the corresponding normalized power (i.e. time-dependent power divided by constant input laser power), including the absorbed part and lost parts (Fig. 3A-F). The simulation result presents four distinct regimes of this transient process: (i) melting and melt pool formation, (ii) vapor depression and keyhole growth, (iii) keyhole fluctuation, and (iv) quasi-steady state (Fig. 3A and 3B). These four regimes have been observed experimentally (2). The time-resolved variations in absorbed power arise because of transient keyhole dynamics. Once the peak temperature is higher than the boiling temperature of the material, the absorbed power rapidly increases as the keyhole depression deepens because the multireflection of the laser beam between the walls of the depression. After this transition, the keyhole walls fluctuate strongly, which leads to fluctuations in all the normalized powers. The lower-frequency fluctuations on the order of $10^{4} \mathrm{~Hz}$ then gradually disappear, while a higher-frequency fluctuation on the order of $10^{6} \mathrm{~Hz}$ still exists. The magnitude of the higher-frequency fluctuation for each normalized power in the quasi-steady-state is recorded as the error bar showing in Fig. $3 \mathrm{~A}-\mathrm{B}$, and $3 \mathrm{G}-\mathrm{H}$. We identify a scaling law of the process-induced absorption using 30 multiphysics simulation cases. This scaling law is validated using experimentally measured absorptivity (3) (marked on the Fig. 3G). The governing parameters include the Keyhole number $\mathrm{Ke}_{\mathrm{m}}$ with minimum absorptivity $\eta_{m}$ of each material, i.e., absorptivity on flat melt surface (3), 30 and a normalized diffusion length $\mathrm{L}_{\mathrm{d}}^{*}$ (Supplementary Text). The normalized absorbed power (absorptivity) can be expressed as

$$
\begin{gathered}
\eta=\eta_{\text {absor }}=0.7\left(1-e^{\left.-0.6 \mathrm{Ke}_{\mathrm{m}} \mathrm{L}_{\mathrm{d}}^{*}\right),}\right. \\
\mathrm{Ke}_{\mathrm{m}} \mathrm{L}_{\mathrm{d}}^{*}=\frac{\eta_{m} P}{\left(T_{l}-T_{0}\right) \pi \rho C_{p} \sqrt{\alpha V_{s} r_{0}^{3}}} \cdot \sqrt{\frac{\alpha}{V_{s} r_{0}}}=\frac{\eta_{m} P}{\left(T_{l}-T_{0}\right) \pi \rho C_{p} V_{s} r_{0}^{2}} .
\end{gathered}
$$



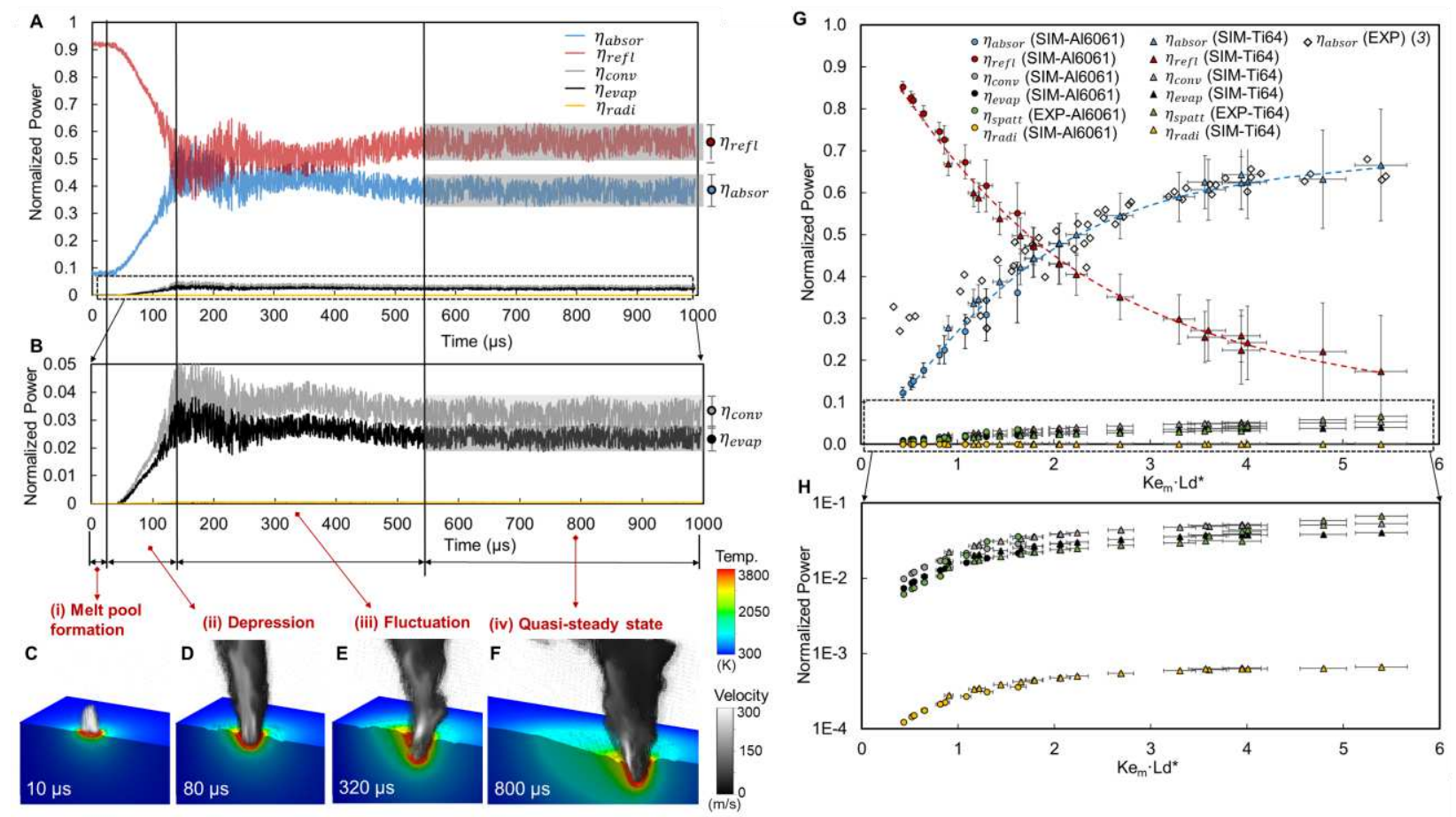

Fig. 3. Simulation of the evolution of normalized powers. (A) Transient normalized powers for a case of Ti6Al4V (laser power is $210 \mathrm{~W}$ and scan speed is $0.4 \mathrm{~m} / \mathrm{s}$.), including normalized absorbed power $\eta_{a b s o r}$, reflected power $\eta_{\text {refl }}$, convected power $\eta_{\text {conv }}$, evaporated power $\eta_{\text {evap }}$, and radiated power $\eta_{\text {radi }}$. Four regimes are classified based on different features of the curves. (B) Magnification plot of transient normalized powers ranging from 0 to 0.05 , to highlight the convection and evaporation terms. (C-F) Illustrative simulated keyhole morphologies and temperature fields showing the free surface and longitudinal cross-section, and vapor velocity fields at different time. (G) Normalized powers with different laser powers, scan speeds, laser spot sizes, and two materials (Ti6Al4V and 6061 Aluminum alloy) (Data S2). Experimental absorptivity data including three different materials (Ti6Al4V, Stainless steel 316 and Inconel 718) from the literature (3) (marked as hollow diamonds) is used to validate the model prediction. The discrepancy at low normalized power is because the simulated material (A16061) has much lower minimum absorptivity than the experimentally measured materials. (H) Magnification logarithm plot of normalized powers ranging from 0 to 0.1 .

The scaling parameter $\mathrm{Ke}_{\mathrm{m}} \mathrm{L}_{\mathrm{d}}^{*}$ represents a product of the volumetric energy density of the laser heat source and the inverse of the sensible heat of melting. The validated multiphysics model can provide a detailed breakdown of the energy transfer terms, which cannot be directly measured experimentally. We conclude that the absorbed and reflected powers dominate the laser absorption since the sum of normalized powers including convection, evaporation, radiation, and spattering are around 0.1 . Radiative energy loss is neglectable. This rationalizes the fact that the vaporization-related parameters, such as boiling point and latent heat of vaporization, are not necessarily included in the scaling laws. The spattering power is measured from x-ray imaging series for bare plate experiments (Supplementary Text). The spattering is more frequent in laser powder bed fusion process because of the particle entrainment effect (23), which might lead to higher spattering power loss.

Furthermore, we quantify inherent correlation between keyhole stability and porosity formation in metal 3D printing for the first time. We analyze the previously reported printinginduced porosity data (Fig. 4). A strikingly simple scaling law defines a two-dimensional pattern that retains descriptive capabilities for porosity data collected from six independent studies (6, 17, 24-27) spanning ten process parameter sets and material properties. This scaling law is expressed as 


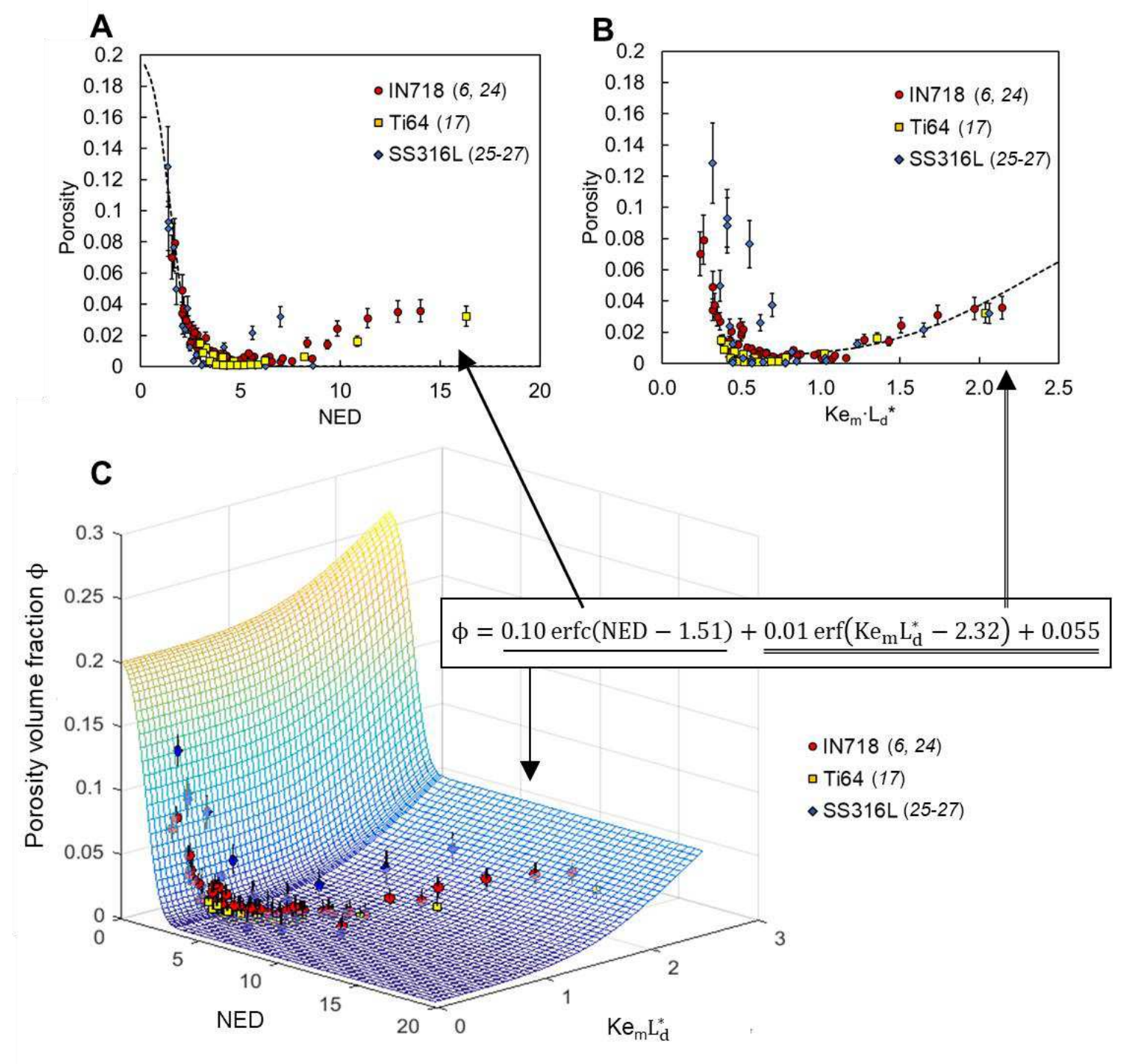

Fig. 4. Two-dimensional (2D) scaling law for volume fraction of porosity. (A) The first term of the scaling law associated with the normalized energy density NED, which quantifies porosity formed via the lack of fusion process. (B) The second and the constant terms of the scaling law associated with the product of Keyhole number, $\mathrm{Ke}_{\mathrm{m}}$, and normalized diffusion length $L_{d}^{*}$, which quantifies the keyhole porosity. (C) 2D scaling law combining both lack-of-fusion and keyhole porosity. Methods of measuring the porosity and computing error bars are provided in the literature $(6,17,24-27)$. This reduced function space is much more easily visualized and interpreted than the original high-dimensional problem in which the porosity is controlled by 10 parameters. By making it easy to visualize, we enable more intuitive optimization and use.

$$
\phi=0.10 \operatorname{erfc}(\mathrm{NED}-1.51)+0.01 \operatorname{erf}\left(\mathrm{Ke}_{\mathrm{m}} \mathrm{L}_{\mathrm{d}}^{*}-2.32\right)+0.055
$$

where $\phi$ describes the volume fraction of the porosity. A Gaussian error function, i.e., $\operatorname{erf}(x)=\frac{1}{\sqrt{\pi}} \int_{-x}^{x} e^{-t^{2}} d t$, and its complementary function, defined as $\operatorname{erfc}(x)=1-\operatorname{erf}(x), \operatorname{completely}$ describes the data, which implies that the origin of porosity formation might be related to specific Gaussian random processes. Two dimensionless numbers dominate the low-dimensional pattern with good accuracy (coefficient of determination, $R^{2}$, is 0.92 ). The first dimensionless number is the well-known normalized energy density (NED) (4-6), defined by 


$$
\mathrm{NED}=\left(\frac{\eta_{m} P}{V_{s} H L}\right)\left(\frac{1}{\rho C_{p}\left(T_{l}-T_{0}\right)}\right)
$$

where $H$ is the hatch spacing and $L$ is the powder bed thickness in laser powder-bed fusion process. The normalized energy density represents the ratio of effective energy density within the powder bed to sensible heat of melting. Interestingly, the product of the Keyhole number $\mathrm{Ke}_{\mathrm{m}}$ (with minimum absorptivity $\eta_{m}$ ) and the normalized diffusion length $L_{d}^{*}$ appears again as the second independent parameter, despite the fact that this porosity model is completely independent from the previous case of keyhole dynamics. Although powder effects affect the laser energy absorptivity, especially in the low-power range (10), the Keyhole number does not include the layer thickness of the powder bed $L$ because our aim is to generate a simplified relationship, and the existence of a powder bed has only a small effect on the keyhole. This is consistent with experimental observations (2). The appearance of the Keyhole number in these seemingly unrelated problems (keyhole morphology and porosity) implies that the porosity generation is analogous to keyhole formation and will not appear below a critical Keyhole number.

The first term of the scaling law for porosity is related only to the NED. This term fits data with low NED, which is a regime characterized by lack-of-fusion defects (Fig. 4A). When NED exceeds the critical value (approximately 5), lack-of-fusion porosity can be avoided and the efficacy of NED as a predictor of porosity decreases. Meanwhile, the second term and the constant part of the scaling law begin to represent the observed data well (Fig. 4B). Since the Keyhole number $\mathrm{Ke}_{\mathrm{m}}$ and normalized diffusion length $\mathrm{L}_{\mathrm{d}}^{*}$ are determinant parameters here, this part of the scaling law can be understood as higher input energies related to sensible heat of melting resulting in keyhole porosity, and the scaling law quantifies the effect of parameters. The combination of the two mechanisms as a 2D scaling law is visualized (shown as a mesh surface) along with experimental data points (colored dots) in Fig. 4C. The newly discovered scaling law (Equation 8 and Fig. 4C) is revolutionary as compared with the traditional NED description (Equation 9 and Fig. 4A) because of its universality. Equation 8 captures minimal porosity (near full density) conditions for three different AM materials and is able to be extended for more materials, and therefore provides a simple transition rule that translates optimal process parameters from one material (or existing materials) to another (or new materials). However, the traditional NED description (Fig. 4A) captures differences in near-full density conditions for different materials but fails to provide any relation between them. Thus, it is not helpful to approximate the near full density condition for a new material based on the existing data.

We identify simple scaling laws for keyhole aspect ratio and its variability using real time X-ray imaging and multiphysics modeling. An inherent correlation between keyhole stability and porosity formation in metal 3D printing is also quantified for the first time. The keyhole dynamics embedded by these compact scaling laws provides a new avenue to quantify processinduced phenomena in metal 3D printing. These concise laws are able to reduce complex, highly multivariate problem spaces into descriptions involving just a few physically interpretable parameters. The dominant laws for keyhole stability and porosity reveal dynamics underlying the laser-metal interaction processes, which can potentially lead to quantitative predictive models for controlling defects generation in metal 3D printing. The reduction of high dimensional parameter space means that fewer experiments will be required to determine optimal processing conditions for new materials, and thus ease the Edisonian burden endemic among current metal 3D printing practitioners. 


\section{Methods}

Materials

We manufactured $50 \mathrm{~mm}$ by $3 \mathrm{~mm}$ by $0.75 \mathrm{~mm}$ samples of aluminum (Al6061, McMasterCarr, USA) and $50 \mathrm{~mm}$ by $3 \mathrm{~mm}$ by $0.5 \mathrm{~mm}$ samples of stainless steel (SS316, McMaster-Carr, USA) from as-received plates using conventional manufacturing methods (cutting and milling). The samples were polished on all sides before being loaded into the vacuum chamber at the beamline.

\section{$\underline{\text { Selective laser melting apparatus }}$}

We built a custom selective laser melting apparatus by integrating an ytterbium fiber laser (YLR-500-AC, IPG, USA), a galvo laser scanner (IntelliSCANde30, SCANLAB GmbH., Germany), a vacuum chamber, and multiple translational motor stages. Ar gas is filled into the chamber to prevent the potential oxidation of the metals. The laser wavelength was $1070 \mathrm{~nm}$ and the maximum power was $540 \mathrm{~W}$. The laser source was operated in single mode, providing a Gaussian beam profile. At the focal plane, the laser spot size was $\approx 50 \mu \mathrm{m}$. In this study, samples were positioned at a certain distance below the focal plane to achieve larger laser spot sizes on sample surface $(1.5 \mathrm{~mm}$ for a spot size of $84 \mu \mathrm{m}, 1.8 \mathrm{~mm}$ for $93 \mu \mathrm{m}, 2.5 \mathrm{~mm}$ for $114 \mu \mathrm{m}$, and 3.5 $\mathrm{mm}$ for $144 \mu \mathrm{m})$. Uncertainty of the laser spot size on sample is about $\pm 5 \mu \mathrm{m}$. Single track laser melting experiments were performed on the samples under various laser powers (208 $\mathrm{W}$ to 520 $\mathrm{W})$ and scan speeds $(0.3 \mathrm{~m} / \mathrm{s}$ to $1.2 \mathrm{~m} / \mathrm{s})$.

\section{High-speed x-ray imaging}

The high-speed $\mathrm{x}$-ray imaging experiments were performed at beamline 32-ID-B of the Advanced Photon Source at Argonne National Laboratory. A short period $(18 \mathrm{~mm})$ undulator with the gap set to $12 \mathrm{~mm}$ was used to generate polychromatic x-rays with the first harmonic energy centered at $24.7 \mathrm{keV}$. The x-rays were allowed to pass through the sample while the laser was traversing across the top surface. The propagated $\mathrm{x}$-ray signal was converted to visible light using a LuAG:Ce scintillator (100 $\mu \mathrm{m}$ thickness) and recorded with a high-speed camera (Photron FastCam SA-Z, USA) after passing a $45^{\circ}$ reflection mirror, a relay lens, and a $10 \times$ objective lens. The nominal spatial resolution of the imaging system was $1.93 \mu \mathrm{m} / \mathrm{pixel}$. We recorded high-speed x-ray images at frame rates between 20,000 and 50,000 frames per second with exposure times between $1 \mu$ s and $40 \mu$ s, with higher exposure times used for stainless steel samples. A series of delay generators were used to trigger the x-ray shutters, laser system, and high-speed camera sequentially. More details of the high-speed x-ray imaging experiments on laser additive manufacturing are provided elsewhere $(12,13)$.

\section{Image processing and quantification}

The images of keyholes and melt pools were processed and analyzed using ImageJ (28). Each image stack includes a time series of images. For each we first duplicated the original image stack (A) to create an identical stack (B). Second, we duplicated the first slice of stack A and the last slice of the stack B. Third, we divided image stack A pixel-wise and slice-wise for each slice by stack B to reduce background noise and increase contrast of the interesting structure features. Fourth, we used a despeckling median filter to further eliminate speckle noise. Finally, we adjusted the brightness and contrast manually to enhance contrast and ease visualization and dimension measurement. We quantified the size of the melt pool and keyhole based on contours of attenuation contrast. This involved manual inspection and measurement of 
each image in all the image stacks in ImageJ, recording the XY coordinates describing length and depth of the melt pool and keyhole for each image. The maximum, minimum, mean and standard deviation for each stack were calculated.

\section{References and Notes:}

1. J. H. Martin, B. D. Yahata, J. M. Hundley, J. A. Mayer, T. A. Schaedler, T. M. Pollock, 3D printing of high-strength aluminum alloys. Nature 549, 365 (2017).

2. R. Cunningham, C. Zhao, N. Parab, C. Kantzos, J. Pauza, K. Fezzaa, T. Sun, A. D. Rollett, Keyhole threshold and morphology in laser melting revealed by ultrahigh-speed $\mathrm{x}$-ray imaging. Science 363, 849-852 (2019).

3. J. Ye, S. A. Khairallah, A. M. Rubenchik, M. F. Crumb, G. Guss, J. Belak, M. J. Matthews, Energy Coupling Mechanisms and Scaling Behavior Associated with Laser Powder Bed Fusion Additive Manufacturing. Adv. Eng. Mater. 16, 1900185 (2019).

4. A. M. Rubenchik, W. E. King, S. S. Wu, Scaling laws for the additive manufacturing. J. Mater. Process. Tech. 257, 234-243 (2018).

5. R. Fabbro, M. Dal, P. Peyre, F. Coste, M. Schneider, V. Gunenthiram, Analysis and possible estimation of keyhole depths evolution using laser operating parameters and material properties. J. Laser Appl. 30, 032410 (2018).

6. Z. Wang, M. Liu, Dimensionless analysis on selective laser melting to predict porosity and track morphology. J. Mater. Process. Technol. 273, 116238 (2019).

7. S. A. Khairallah, A. T. Anderson, A. Rubenchik, W. E. King, Laser powder-bed fusion additive manufacturing: Physicsof complex melt flow and formation mechanisms of pores, spatter, and denudation zones. Acta Mater. 108, 36-45 (2016).

8. C. Zhao, Q. Guo, X. Li, N. Parab, K. Fezzaa, W. Tan, L. Chen, T. Sun, Bulk-explosioninduced metal spattering during laser processing. Phys. Rev. X 9, 021052 (2019).

9. T. DebRoy, H.L.Wei, J.S. Zuback, T. Mukherjee, J.W. Elmer, J.O. Milewski, A.M. Beese, A.D. Wilson-Heid, A. De and W. Zhang, Additive manufacturing of metallic componentsprocess, structure and properties. Prog. Mater. Sci, 92, 112-224 (2018).

10. S.A. Khairallah, A.A. Martin, J.R. Lee, G. Guss, N.P. Calta, J.A. Hammons, M.H. Nielsen, K. Chaput, E. Schwalbach, M.N. Shah and M.G. Chapman, Controlling interdependent meso-nanosecond dynamics and defect generation in metal 3D printing. Science, 368, 660665 (2020).

11. D. T. Swift-Hook, A. E. F. Gick, Penetration welding with lasers. Weld. J. 52, 492s-499s (1973).

12. C. Zhao, K. Fezzaa, R.W. Cunningham, H. Wen, F. De Carlo, L. Chen, A.D. Rollett, T. Sun, Real-time monitoring of laser powder bed fusion process using high-speed X-ray imaging and diffraction. Sci. Rep. 7, 1-11 (2017).

13. N.D. Parab, C. Zhao, R. Cunningham, L.I. Escano, K. Fezzaa, W. Everhart, A.D. Rollett, L. Chen, T. Sun, Ultrafast X-ray imaging of laser-metal additive manufacturing processes. $J$. Synchrotron Radiat. 25, 1467-1477 (2018). 
14. C.L.A. Leung, S. Marussi, R.C. Atwood, M. Towrie, P.J. Withers, P.D. Lee, In situ X-ray imaging of defect and molten pool dynamics in laser additive manufacturing. Nat. Commun. 9, 1-9 (2018).

15. N.P. Calta, J. Wang, A.M. Kiss, A.A. Martin, P.J. Depond, G.M. Guss, V. Thampy, A.Y.

Fong, J.N. Weker, K.H. Stone, C.J. Tassone, An instrument for in situ time-resolved X-ray imaging and diffraction of laser powder bed fusion additive manufacturing processes. Rev. Sci. Instrum. 89, 055101 (2018).

16. S.M.H. Hojjatzadeh, N.D. Parab, W. Yan, Q. Guo, L. Xiong, C. Zhao, M. Qu, L.I. Escano, X. Xiao, K. Fezzaa, W. Everhart, Pore elimination mechanisms during 3D printing of metals. Nat. Commun. 10, 1-8 (2019).

17. G. Kasperovich, J. Haubrich, J. Gussone, G. Requena, Correlation between porosity and processing parameters in TiAl6V4 produced by selective laser melting. Mater. Des. 105, 160-170 (2016).

18. M. Tang, P. C. Pistorius, Oxides, porosity and fatigue performance of alsi10mg parts produced by selective laser melting. Int. J. Fatigue 94, 192-201 (2017).

19. C. Weingarten, D. Buchbinder, N. Pirch, W. Meiners, K. Wissenbach, R. Poprawe, Formation and reduction of hydrogen porosity during selective laser melting of AlSi10Mg. $J$. Mater. Process. Technol. 221, 112-120 (2015).

20. G. I. Barenblatt, Scaling (Cambridge University Press, Cambridge, UK, 2003).

21. E. Buckingham, On physically similar systems; illustrations of the use of dimensional equations. Phys. Rev. 4, 345 (1914).

22. J. R. Koza, Genetic programming (MIT Press, Cambridge, MA,1992).

23. Y. A. Mayi, M. Dal, P. Peyre, M. Bellet, C. Metton, C. Moriconi, R. Fabbro, Laser-induced plume investigated by finite element modelling and scaling of particle entrainment in laser powder bed fusion. J. Phy. D: Appl. Phys. 53, 075306 (2019).

24. P. Kumar, J. Farah, J. Akram, C. Teng, J. Ginn, M. Misra, Influence of laser processing parameters on porosity in Inconel 718 during additive manufacturing. Inter. J. of Adv. Manuf. Tech. 103, 1497-1507 (2019).

25. J. A. Cherry, H. M. Davies, S. Mehmood, N. P. Lavery, S. G. Brown, J. Sienz, Investigation into the effect of process parameters on microstructural and physical properties of $316 \mathrm{~L}$ stainless steel parts by selective laser melting. Int. J. Adv. Manuf. Technol. 76, 869-879 (2015).

26. A. Leicht, M. Rashidi, U. Klement, E. Hryha, Effect of process parameters on the microstructure, tensile strength and productivity of 316L parts produced by laser powder bed fusion. Mater. Charact. 159, 110016 (2020).

27. J. C. Simmons, X. Chen, A. Azizi, M. A. Daeumer, P. Y. Zavalij, G. Zhou, S. N. Schiffres, Influence of processing and microstructure on the local and bulk thermal conductivity of selective laser melted 316L stainless steel. Addit. Manuf. 32, 100996 (2020).

28. T. Ferreira, W. Rasband, https://imagej.nih.gov/ij/ (2012).

29. Z. Gan, G. Yu, X. He, S. Li, Numerical simulation of thermal behavior and multicomponent mass transfer in direct laser deposition of Co-base alloy on steel. Int. J. Heat Mass Transf. 104, 28-38 (2017). 
30. A. A. Samokhin, Effect of laser radiation on absorbing condensed matter. Proceedings of the Institute of General Physics Academy of Sciences of the USSR. Series Editor: AM Prokhorov, 13, 203 (1990).

31. D. Kondepudi, I. Prigogine, Modern thermodynamics: from heat engines to dissipative structures (John Wiley \& Sons, 2014).

32. S. W. Han, J. Ahn, S. J. Na, A study on ray tracing method for CFD simulations of laser keyhole welding: progressive search method. Weld. World 60, 247-258 (2016).

33. S. Pang, L. Chen, J. Zhou, Y. Yin, T. Chen, A three-dimensional sharp interface model for self-consistent keyhole and weld pool dynamics in deep penetration laser welding. J. Phys. $D$ 44, 025301 (2010).

34. Ansys Inc, ANSYS FLUENT theory guide: Release 2020 R1 (Canonsburg, PA, 2020).

35. C. W. Hirt, B. D. Nichols, Volume of fluid (VOF) method for the dynamics of free boundaries. J. Comput. Phys. 39, 201-225 (1981).

36. W. E. King, A. T. Anderson, R. M. Ferencz, N. E. Hodge, C. Kamath, S. A. Khairallah, A. M. Rubenchik, Laser powder bed fusion additive manufacturing of metals; physics, computational, and materials challenges. Appl. Phys. Rev. 2, 041304 (2015).

37. A. Arora, G. G. Roy, T. DebRoy, Unusual wavy weld pool boundary from dimensional analysis. Scr. Mater. 60, 68-71 (2009).

38. J. J. Valencia, N. Q. Peter, Thermophysical properties. (2013).

Acknowledgments: We gratefully acknowledge the computing resources provided on Bebop, a high-performance computing cluster operated by the Laboratory Computing Resource Center at Argonne National Laboratory. We also thank the Center for Hierarchical Materials Design (CHiMaD), in particular, our ongoing work with Lyle E. Levine has been synergistic. Funding: W.K.L., Z.G., O.L.K., and L.F. were supported by the National Science Foundation (NSF) through grants CMMI-1762035, CMMI-1934367, and CMMI-1646592. O.L.K. acknowledges support through the NSF Graduate Research Fellowship under Grant No. DGE-1324585. N.D.P., C.Z., and T.S. would like to acknowledge Laboratory Directed Research and Development (LDRD) funding from Argonne National Laboratory, provided by the Director, Office of Science, of the U.S. Department of Energy under Contract No. DE-AC02-06CH11357; the work by O.G.H. was performed under financial assistance award 70NANB14H012 from U.S. Department of Commerce, National Institute of Standards and Technology as part of the CHiMaD. This research used resources of the Advanced Photon Source; a U.S. Department of Energy (DOE) Office of Science User Facility operated for the DOE Office of Science by Argonne National Laboratory under Contract No. DE-AC02-06CH11357. Author contributions: O.G.H., W.K.L. Z. G. and O.L.K. procured funding and supervised the project. Z.G., O.L.K. and O.G.H. analyzed the results and wrote the first manuscript. N.D.P., C.Z. and T.S. designed and conducted the experiments. Z.G. implemented the multiphysics modeling and dimensional analysis. L.F. conducted post-processing of x-ray images; Competing interests: Authors declare no competing interests. Data and materials availability: All data is available in the main text or the supplementary materials.

\section{Supplementary Materials:}

Figures S1-S9

Tables S1-S3 
Movies S1-S11

Data S1-S2 


\section{Figures}
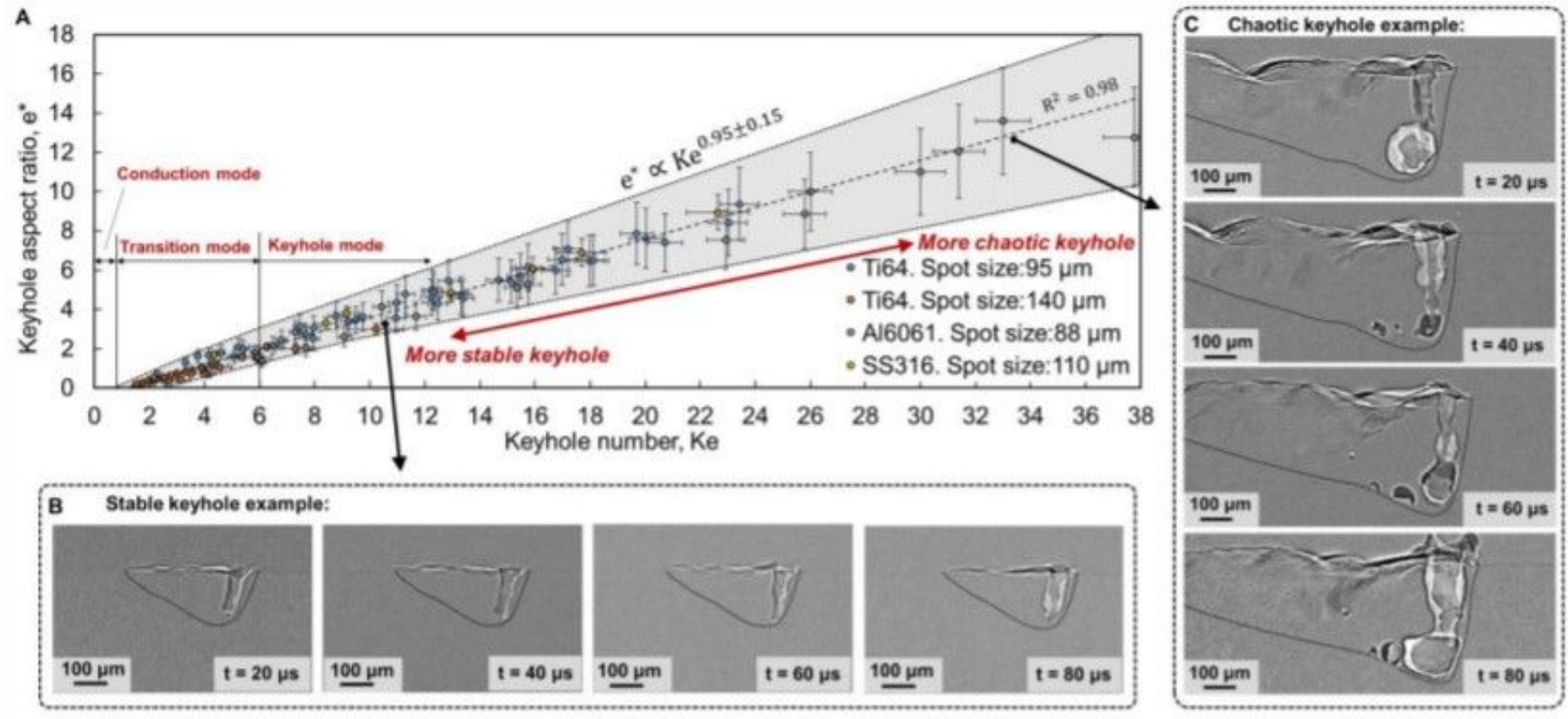

\section{Figure 1}

One-dimensional law for keyhole aspect ratio controlled by the Keyhole number. (A) Identified scaling law, which is universal because all the data collapse to a single curve, even though they correspond to various laser power (100-520 W), scan speed (0.3-1.2 m/s), laser spot size $(44-70 \mu \mathrm{m})$, and three different materials (Ti-6Al-4V (Ti64) (2), Aluminum alloy 6061 (Al6061) and Stainless Steel 316 (SS316)). Timedependent keyhole depth is measured from high-speed $\mathrm{x}$-ray images at a $20 \mu \mathrm{s}$ interval for each process condition. The maximum and minimum of keyhole aspect ratio during the time period when the laser scans $2 \mathrm{~mm}$ length at the middle of the sample are marked as vertical error bars. Horizonal error bars account for uncertainties of the process parameters and material properties. (B) Operando $x$-ray image series with a $20 \mu$ s interval showing the keyhole and melt pool morphologies at stable keyhole region of Al6061. Laser power is $416 \mathrm{~W}$ and scan speed is $0.6 \mathrm{~m} / \mathrm{s}$. (C) Operando x-ray images showing keyhole morphologies at chaotic keyhole region of Al6061. Laser power is $520 \mathrm{~W}$ and scan speed is $0.3 \mathrm{~m} / \mathrm{s}$. Fusion boundary (outlined by the black dashed line) can be identified by the x-ray imaging because of the density difference inside and outside the fusion region. Although the x-ray experiments are conducted on a bare plate, a previous study reports that the keyhole depth does not change significantly by adding a thin layer of powder (2). 




Figure 2

Comparison between experimental data and multiphysics simulations with various Keyhole number. (AD) Operando x-ray images of the scanning laser melting of Al6061 at steady state. (E-H) Multiphysics modeling showing temperature contour at the longitudinal cross-section and arrow field of vapor velocity above the substrate (the length of the arrow is proportional to the magnitude of velocity at the specific point). The simulated high-speed vapor jet ( $200 \mathrm{~m} / \mathrm{s}$ ) impacts keyhole interface which is one of the sources driving the fluctuation of the keyhole and energy loss due to convection
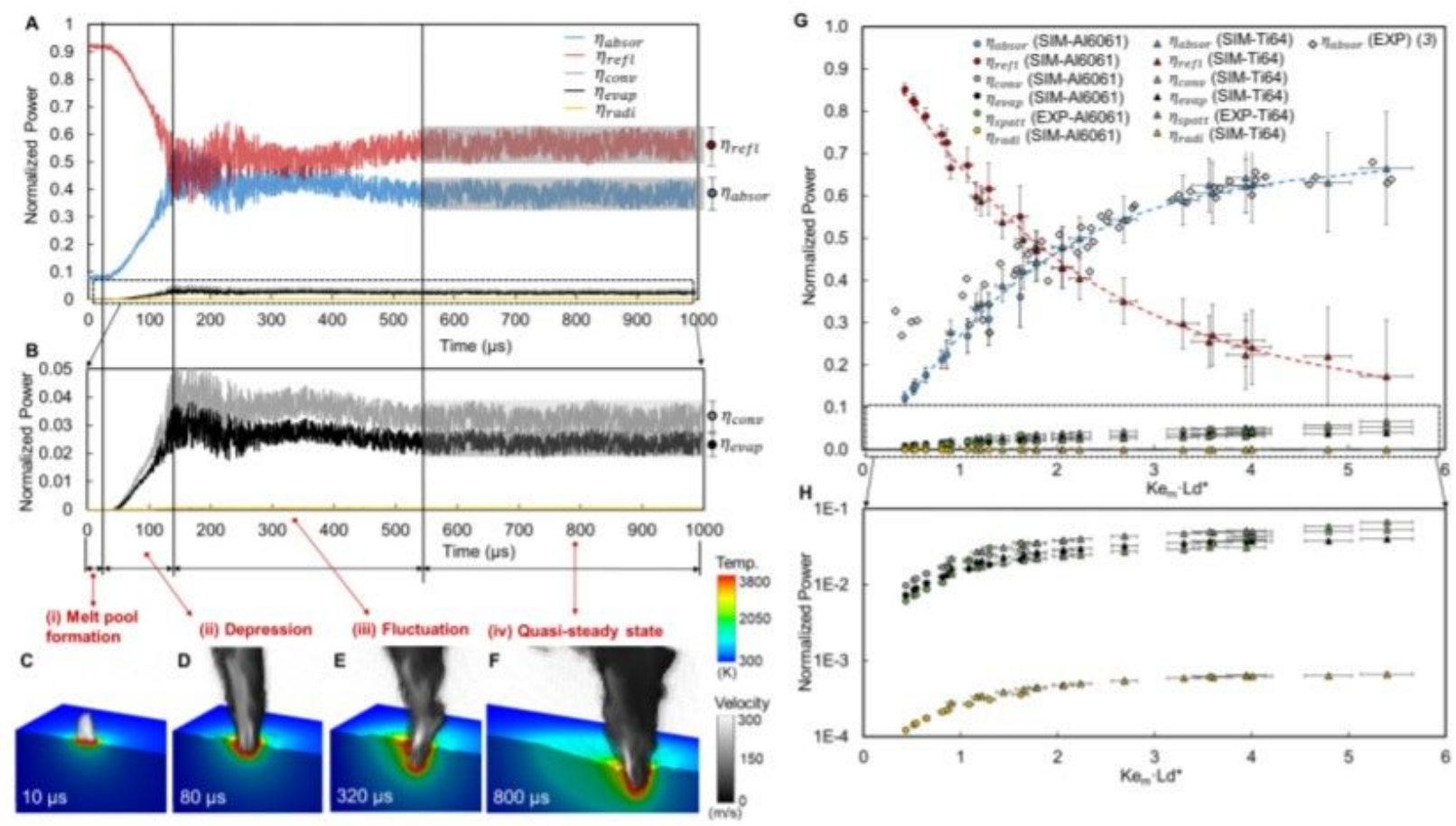

Figure 3 
Simulation of the evolution of normalized powers. (A) Transient normalized powers for a case of Ti6Al4V

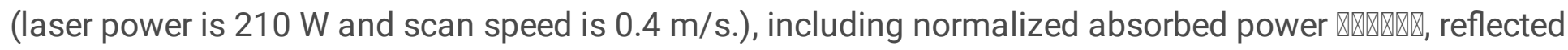

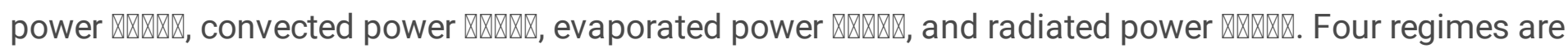
classified based on different features of the curves. (B) Magnification plot of transient normalized powers ranging from 0 to 0.05 , to highlight the convection and evaporation terms. (C-F) Illustrative simulated keyhole morphologies and temperature fields showing the free surface and longitudinal cross-section, and vapor velocity fields at different time. (G) Normalized powers with different laser powers, scan speeds, laser spot sizes, and two materials (Ti6Al4V and 6061 Aluminum alloy) (Data S2). Experimental absorptivity data including three different materials (Ti6Al4V, Stainless steel 316 and Inconel 718) from the literature (3) (marked as hollow diamonds) is used to validate the model prediction. The discrepancy at low normalized power is because the simulated material (Al6061) has much lower minimum absorptivity than the experimentally measured materials. $(\mathrm{H})$ Magnification logarithm plot of normalized powers ranging from 0 to 0.1 .

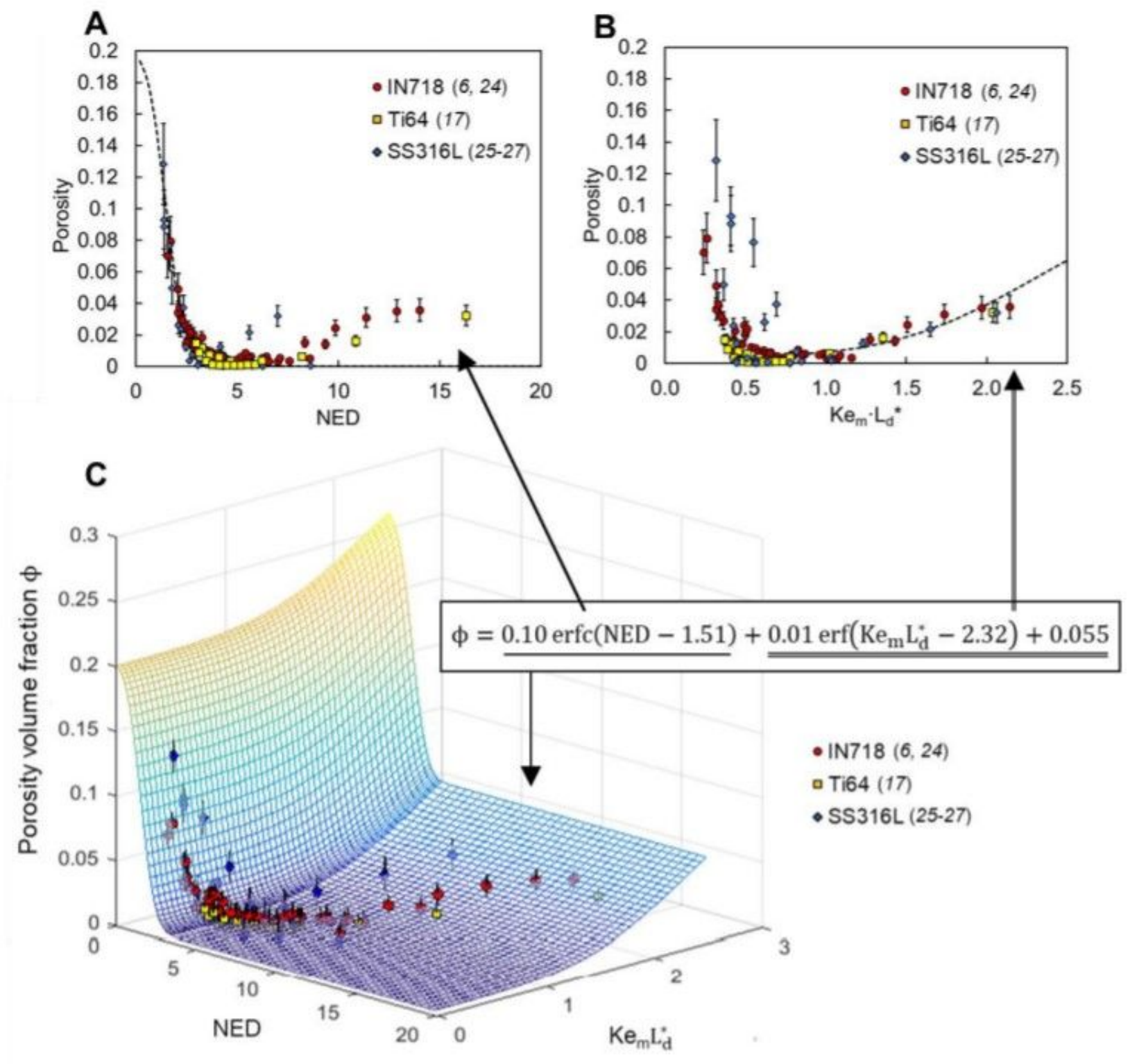




\section{Figure 4}

Two-dimensional (2D) scaling law for volume fraction of porosity. (A) The first term of the scaling law associated with the normalized energy density NED, which quantifies porosity formed via the lack of fusion process. (B) The second and the constant terms of the scaling law associated with the product of Keyhole number, Kem, and normalized diffusion length $\forall \mathbb{Q}$, which quantifies the keyhole porosity. (C) 2D scaling law combining both lack-of-fusion and keyhole porosity. Methods of measuring the porosity and computing error bars are provided in the literature $(6,17,24-27)$. This reduced function space is much more easily visualized and interpreted than the original high-dimensional problem in which the porosity is controlled by 10 parameters. By making it easy to visualize, we enable more intuitive optimization and use.

\section{Supplementary Files}

This is a list of supplementary files associated with this preprint. Click to download.

- SupplementaryMaterialsrevised.pdf 